\title{
Deciphering Kinetic Information from Single-Molecule FRET Data That Show Slow Transitions
}

\author{
Sung Eun Kim, ${ }^{\dagger}$ Il-Buem Lee, ${ }^{\dagger}$ Changbong Hyeon, ${ }^{\ddagger}$ and Seok-Cheol Hong* ${ }^{\dagger},+$ \\ ${ }^{\dagger}$ Department of Physics, Korea University, Seoul 136-713, Republic of Korea \\ ${ }^{\ddagger}$ Korea Institute for Advanced Study, Seoul 130-722, Republic of Korea
}

Supporting Information

ABSTRACT: Single-molecule FRET is one of the most powerful and widely used biophysical techniques in biological sciences. It, however, often suffers from limitations such as weak signal and limited measurement time intrinsic to singlemolecule fluorescence measurements. Despite several ameliorative measures taken to increase measurement time, it is nearly impossible to acquire meaningful kinetic information on a molecule if conformational transitions of the molecule are ultraslow such that transition times $\left(\langle\tau\rangle_{\text {orig }}\right)$ are comparable to or longer than measurement times $(\delta t)$ limited by the finite lifetime of fluorescent dye. Here, to extract a reliable and

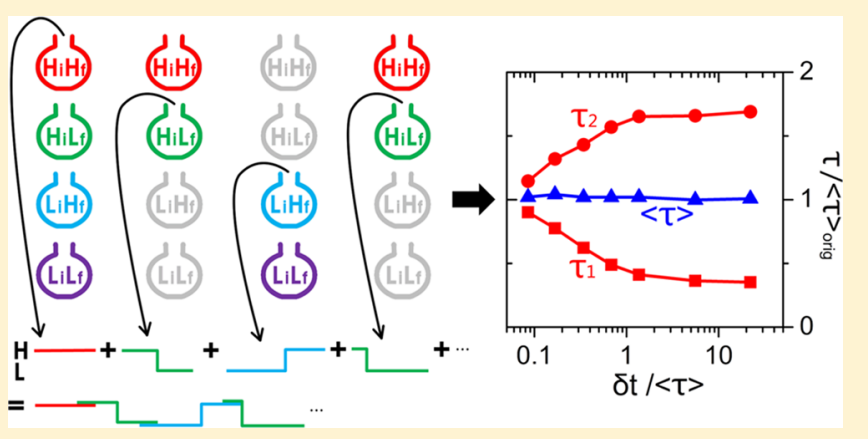
accurate mean transition time from a series of short time traces with ultraslow kinetics, we suggest a scheme called sHaRPer (serialized Handshaking Repeated Permutation with end removal) that concatenates multiple time traces. Because data acquisition frequency $f$ and measurement time $(\delta t)$ affect the estimation of mean transition time $(\langle\tau\rangle)$, we provide mathematical criteria that $f$, $\delta t$, and $\langle\tau\rangle$ should satisfy to make $\langle\tau\rangle$ close enough to $\langle\tau\rangle_{\text {orig. }}$ Although application of the sHaRPer method has a potential risk of distorting the time constants of individual kinetic phases if the data are described with kinetic partitioning, we also provide criteria to avoid such distortion. Our sHaRPer method is a useful way to handle single-molecule data with slow transition kinetics. This study provides a practical guide to use sHaRPer.

\section{INTRODUCTION}

Single-molecule fluorescence resonance energy transfer (smFRET) is one of the most popular and versatile biophysical techniques in the field of biological sciences. ${ }^{1-6}$ It shares, however, the same limitations with other single-molecule fluorescence techniques such as weak signal and limited measurement time due to photobleaching of dyes. Photostability of fluorescent dyes is essential for long measurement times in fluorescence measurements. Several oxygen scavenging methods $^{7-9}$ have been developed over the years to minimize undesirable oxidation of dye molecules because photobleaching of dyes seriously compromises the advantage of fluorescence techniques. Owing to the development of oxygen scavenging methods, measurement times of a few minutes are routinely obtained. ${ }^{10,11}$ While such an innovative improvement in measurement times as well as recent theoretical advances in extracting the maximal information from single molecule trajectories ${ }^{12,13}$ has been made, it is still difficult to obtain reliable kinetic information from experimental data if the dynamics is too slow compared with the measurement times.

We recently developed the concatenation methods to acquire accurate kinetic information from systems with ultraslow kinetics as a remedy to the aforementioned issue. We provide a comprehensive description on a scheme named sHaRPer, which refines the previously developed concatenation methods (HaRP and sHaRP). ${ }^{10}$ We systematically address the accuracy of average kinetic information using sHaRPer for experimental parameters that can be tuned. For data described with kinetic partitioning ${ }^{1,14,15}$ to which the application of sHaRPer could still distort the time constants of the individual phases, we elaborate the minimal requirement to avoid such distortion. This paper is a practical reference of the sHaRPer for the kinetic analysis of time trajectories with ultraslow transitions.

\section{METHODS}

sHaRPer (serialized Handshaking Repeated Permutation with end removal) Method. We developed a method named sHaRPer to acquire reliable kinetics of a system. This concatenation method along with the previous ones is particularly useful when the duration $(\delta t)$ of each time-lapse measurement is comparable to or less than the dwell times of states, and thus a usual kinetic analysis is seriously hampered. Here we consider single-molecule time traces that exhibit transitions between two states $(\mathrm{H}$ : folded state, L: unfolded state), but the method can easily be extended beyond two-state systems. In the method, the key idea is to "assume" the ergodicity of the dynamics and to build a very long FRET time trace from a large number of short traces, the duration of which

Received: April 27, 2015

Revised: May 19, 2015

Published: May 19, 2015 


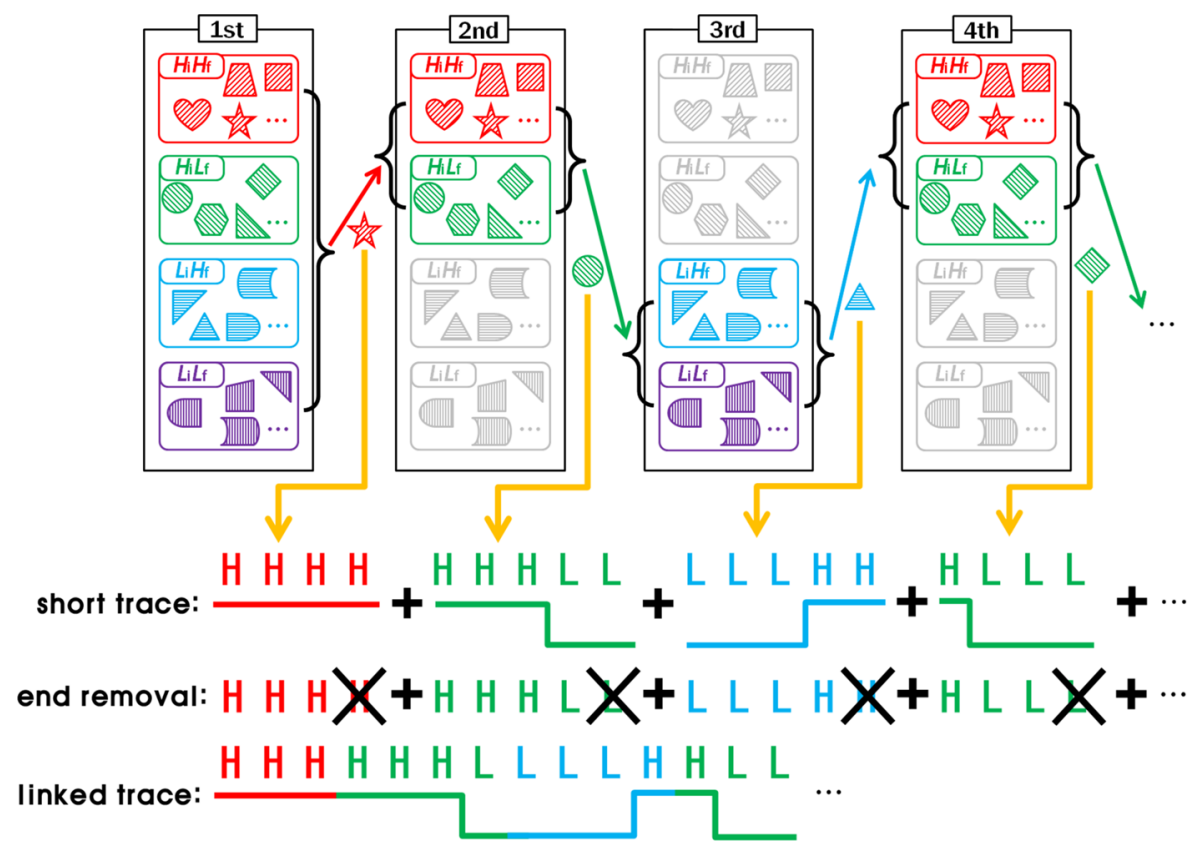

Figure 1. Schematic description of sHaRPer. To create a long trace, we concatenate short traces, which are selected from pools of traces by repeated permutation. The state of the last data point of the composite trace is used to select a next trace to link: the next trace should begin with the same state as the state $(\mathrm{H}$ or $\mathrm{L})$ of the last data point of the preceding trace. Here states $\mathrm{H}$ and $\mathrm{L}$ stand for high- and low-FRET states, respectively. First row: rounds of selection of a short trace from pools of traces via state matching. Each round is depicted as a box. In each box, red/left-hatched, green/right-hatched, sky-blue/horizontal-hatched, and purple/vertical-hatched symbols represent $\mathrm{H}_{\mathrm{i}} \mathrm{H}_{\mathrm{f}}, \mathrm{H}_{\mathrm{i}} \mathrm{L}_{\mathrm{f}}, \mathrm{L}_{\mathrm{i}} \mathrm{H}_{\mathrm{f}}$, and $\mathrm{L}_{\mathrm{i}} \mathrm{L}_{\mathrm{f}}$ traces, respectively. For example, a $\mathrm{H}_{\mathrm{i}} \mathrm{H}_{\mathrm{f}}$ trace has both initial and final states at $\mathrm{H}$ state. Traces are distinguished by different symbols. Curly brackets indicate which pools are considered for trace selection. Irrelevant pools are colored gray. Second row: FRET time trajectory of each selected FRET trace; third row: end removal (shown as $\mathrm{X}$ ) of the sHaRPer method; last row: long linked FRET time trace after sHaRPer.

was limited by dye lifetimes. We first prepare pools of FRET traces, which are classified by the initial and final FRET states of each trace (Figure 1). As illustrated in Figure 1, we randomly select a trace from four different pools $\left(\mathrm{H}_{\mathrm{i}} \mathrm{H}_{\mathfrak{f}}, \mathrm{H}_{\mathrm{i}} \mathrm{L}_{\mathfrak{f}}, \mathrm{L}_{\mathrm{i}} \mathrm{H}_{\mathfrak{f}}\right.$ and $\mathrm{L}_{\mathrm{p}} \mathrm{L}_{\mathrm{f}}$ ) and extend the trace by appending it to the tail of the preceding trace when their FRET states are matched. We iterate this procedure until a long composite trace is built. The previously proposed methods such as HaRP can overestimate $\langle\tau\rangle$ when the trace being concatenated is extremely short, containing only several data points. ${ }^{10}$ The effect of ignoring the possibility that the state changes right after the final data point of the preceding trace becomes more significant for shorter traces. To circumvent the linking mistake inherent in HaRP and thus to avoid an overestimation of $\langle\tau\rangle$ we refine the method by concatenating the first data point of a following trace onto the last data point of the preceding trace or, equivalently, removing the end point of the preceding trace and use the information on the end point only as the guide to link the next trace. (See Figure 1.) We name the refined method sHaRPer. As shown later, the sHaRPer method guarantees the correct estimation of $\langle\tau\rangle$, even in an extremely short trace, accurately preserving the kinetic information on a system as long as the experimental parameters are properly chosen.

\section{RESULTS AND DISCUSSIONS}

Average Dwell Time of a Long Trace Obtained by sHaRPer is Identical to That of the Original Trace. To demonstrate the sHaRPer scheme, we used single-molecule FRET results from the duplex-triplex transition of DNA as experimental data. The same data were used in our previous work. ${ }^{10}$ Although the data exhibit fast kinetics, we use these real data to demonstrate general principle of the sHaRPer method.
To obtain kinetic information, we calculate the survival probability of a state. In the case of the H-to-L (or triplex-toduplex) transition, the survival probability ${ }^{16}$ is described by a double exponential function: $S(t)=A \mathrm{e}^{-t / \tau_{1}}+(1-A) \mathrm{e}^{-t / \tau_{2}}$, and the average dwell time is given by $\tau=\int_{0}^{\infty} S(t) \mathrm{d} t=A \tau_{1}+(1-$ A) $\tau_{2}$.

For the data under consideration, the two time constants are $\tau_{1}=0.57$ and $\tau_{2}=2.5$ and the partitioning factor is $A=0.94$, which leads to the average dwell time $\langle\tau\rangle_{\text {orig }}=0.69 \mathrm{~s}$ (Figure S1a in the Supporting Information (SI)). Now we chop the long original trace into multiple shorter traces (named t-chips) and assume them as traces obtained in real experiments (for example, traces terminated by dye photobleaching). Then, we concatenate the $\mathrm{t}$-chips using sHaRPer. From each long concatenated trace, we obtained the histogram of survival probability and calculated the average dwell time. We repeated the same procedure to get $\langle\tau\rangle$ for 30 different conditions. (See Figure 2a.) For histograms from all 30 traces, see the SI (Figure S1b).

In Figure 2, we constructed a 2D map to show the average dwell time (relative to $\langle\tau\rangle_{\text {orig }}$ ) obtained from a long trace made by $\mathrm{sHaRPer}$ as a function of two parameters: data acquisition frequency $f \times\langle\tau\rangle_{\text {orig }}$ (made to be dimensionless by multiplying $\left.\langle\tau\rangle_{\text {orig }}\right)$ and the number of data points $(\delta p)$ in each trace (related to the duration of time trace). See the SI for details. If the average dwell time obtained from the scheme is close to the true value of $\langle\tau\rangle$, the scheme is judged to be valid. As shown in Figure $2 \mathrm{a},\langle\tau\rangle$ remains unchanged even when $\delta p$ gets very small because the linking mistake is circumvented in sHaRPer.

In contrast with $\delta p$, the slow data acquisition rate $(f)$ overestimates $\langle\tau\rangle$ significantly: as the rate decreases, $\langle\tau\rangle /$ $\langle\tau\rangle_{\text {orig }}$ increases. (See Figure 2a,b.) This is because the dwell 

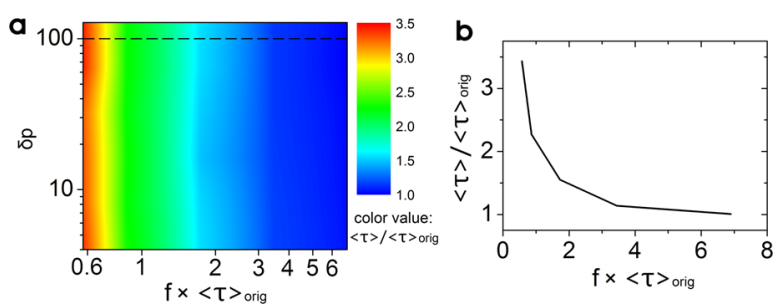

Figure 2. (a) $2 \mathrm{D}$ map of $\langle\tau\rangle \mid\langle\tau\rangle_{\text {orig }}$ in sHaRPer. The $2 \mathrm{D}$ maps are drawn by bilinear interpolation. The number of raw data points used for bilinear interpolation is $30(=6 \times 5)$. The horizontal axis represents the (dimensionless) product of the data acquisition rate $f$ (in $\mathrm{Hz}$ ) and the original average dwell time of the duplex-triplex transition. $f$ covers $0.833-10 \mathrm{~Hz}$ (raw data have five points $(0.833$, $1.25,2.5,5$, and $10 \mathrm{~Hz}$ )) and the average dwell time is fixed to $0.69 \mathrm{~s}$. The vertical axis represents the (dimensionless) number of data points per $\mathrm{t}$-chip, $\delta p$, which is given by the duration of $\mathrm{t}$-chip $(=\delta t)$ times the data acquisition rate $f . \delta p$ covers $4-128$ (raw data have six points $\left(2^{n}\right.$, where $n=2, \ldots, 7))$. (b) $\langle\tau\rangle /\langle\tau\rangle_{\text {orig }}$ versus $f \times\langle\tau\rangle_{\text {orig }}$ for $\delta p=100$. This graph represents the relation of $\langle\tau\rangle$ to $f$ on the dotted line at $\delta p$ $=100$ in panel a. This graph clearly shows that $\langle\tau\rangle \mid\langle\tau\rangle_{\text {orig }}$ remains close to $1(<1.2)$ when $f \times\langle\tau\rangle_{\text {orig }}$ is sufficiently large $(>4)$.

time shorter than the interval of data acquisition cannot be measured accurately. The result shown in Figure 2 recommends that the product of the acquisition rate and $\langle\tau\rangle_{\text {orig }}$ be larger than 4 to estimate $\langle\tau\rangle$ within $20 \%$ accuracy. For this analysis, we did not carry out separate experiments with the indicated acquisition frequencies. Instead, using the data taken at $f_{\mathrm{CCD}}=10 \mathrm{~Hz}$, we kept the first data point in every $N$ data points by omitting all of the other data points in between $(N=$ $1,2,4,8$, and 12 correspond to $f=10,5,2.5,1.25$, and 0.833 $\mathrm{Hz}$, respectively; see SI and Tables $\mathrm{S} 1-\mathrm{S} 3)$. As long as the acquisition rate is high enough $\left(f \times\langle\tau\rangle_{\text {ori }}>4\right)$, the average dwell time is reliably estimated, such that $\langle\tau\rangle \approx\langle\tau\rangle_{\text {orig. }}$.

Kinetic Heterogeneity Can Be Degraded by the sHaRPer Method if the Data Acquisition Time Is Considerably Short. Next, we ask how sHaRPer affects kinetic rates if the system exhibits heterogeneous kinetics, namely, if the observed data obey kinetic partitioning mechanism, described by multiexponentials. To test this, we first synthesized a long time trace whose survival probabilities of the states are described by a double-exponential function with the time constants of $\tau_{1}=2.0 \mathrm{~s}$ and $\tau_{2}=9.6 \mathrm{~s}: S(t)=$ $A \mathrm{e}^{-t / \tau_{1}}+(1-A) \mathrm{e}^{-t / \tau_{2}}$ (Figure $\left.\left.3 \mathrm{a}\right)\right)$ (see SI for details). We then reconstructed a very long trace by concatenating $t$-chips (interval: $\delta t$ ) obtained from the synthetic trace. Figure $3 \mathrm{~b}$ shows that the average dwell time by sHaRPer is little affected by changing $\delta p$, which is consistent with Figure $2 \mathrm{a}$, while the average dwell time by HaRP for small $\delta p$ is considerably larger. In contrast with the average dwell times (Figure $3 \mathrm{~b}$, blue triangle), however, the two dwell times $\left(\tau_{1}\right.$ and $\left.\tau_{2}\right)$ are seriously distorted, converging to a common value between $\tau_{1}$ and $\tau_{2}$, when either $\delta p$ or $\delta t$ becomes small (Figure $3 \mathrm{c}-\mathrm{e}$ ).

It is, however, necessary to examine which factor, the data acquisition rate $(f), \delta t$, or their product, $\delta p=f \times \delta t$, plays a direct and major role in determining the apparent dwell times, $\tau_{1}$ and $\tau_{2}$. As shown in Figure $3 \mathrm{c}$,d, the retrieved values of $\tau_{1}$ and $\tau_{2}$ both display significant deviations from their correct values when $\delta t$ becomes sufficiently short (comparable to or even shorter than $\langle\tau\rangle$ ). The survival probability fitted to a single-exponential function indicates that the averaging process wipes out the kinetic heterogeneity of the original data as $\delta t$ gets shorter. In Figure $3 \mathrm{e}, \tau_{2}$ is affected only slightly because the
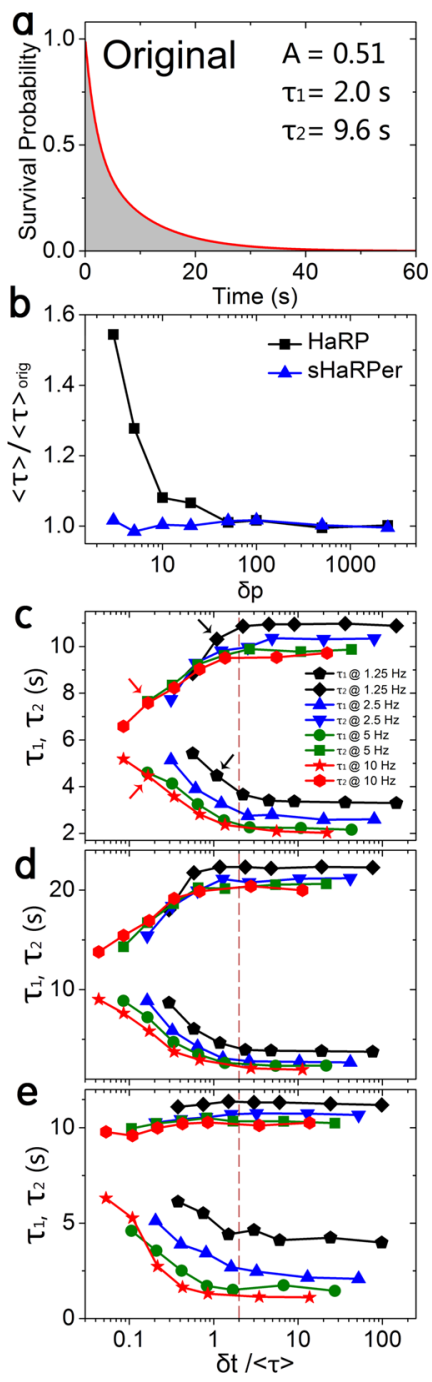

Figure 3. (a) Survival probability of the high FRET state in a simulation trace. To make the simulation trace, we chose $A=0.51, \tau_{1}$ $=2.0 \mathrm{~s}$, and $\tau_{2}=9.6 \mathrm{~s}$. In this case, we get $\langle\tau\rangle_{\text {orig }}=5.8 \mathrm{~s}$. (b) Average dwell times of traces constructed from sHaRPer starting with the simulation trace in panel a. The average dwell time was calculated by population averaging of the two dwell times obtained by doubleexponential fitting of the survival probability. The advantage of sHaRPer over HaRP is obvious because the average dwell times by sHaRPer remain constant for small $\delta p$. (c) For the simulation trace used in panel a, the values for $\tau_{1}$ and $\tau_{2}$ were retrieved by using sHaRPer with $A$ fixed to the original value of $A$. Here we varied the data acquisition frequency $f$ from 1.25 to $10 \mathrm{~Hz}(1.25,2.5,5$, and 10 $\mathrm{Hz}$ ). (d,e) For simulation traces with (d) $\mathrm{A}=0.48, \tau_{1}=2.0 \mathrm{~s}$, and $\tau_{2}=$ $20 \mathrm{~s}$ and (e) $A=0.10, \tau_{1}=1.2 \mathrm{~s}$, and $\tau_{2}=10 \mathrm{~s}$, the values for $\tau_{1}$ and $\tau_{2}$ were retrieved similarly to panel c. Figure labels and conventions in panels $\mathrm{d}$ and e are the same as in panel $\mathrm{c}$. Vertical dotted lines in panels c-e indicate $\delta t /\langle\tau\rangle=2$.

molecules that exhibit slow kinetic phase $\left(\tau_{2}\right)$ occupy a major population (note that its partitioning factor, 1-A, is 0.9), and hence the mixing process by sHaRPer does not affect their kinetics much. Speaking of the effect of $f$, it is reasonable to presume that $f$ would not have any effect on the values of $\tau_{1}$ and $\tau_{2}$ if $f$ is sufficiently large. In that case, any deviation of $\tau_{1}$ and $\tau_{2}$ from their real values originates from short $\delta t$. When $f$ becomes small, both $\tau$ values are overestimated. This is shown in Figure $3 \mathrm{c}$,d. In Figure 3e, the ratio of two $\tau$ values is similar to the ratio in Figure $3 d$, but state 1 is minor to state 2 . Thus, as expected 
from mixing, $\tau_{1}$ goes up quickly toward $\tau_{2}$ while $\tau_{2}$ exhibits relatively small increase. How about $\delta p$ ? One can consider two cases with the same $\delta p$ in Figure $3 \mathrm{c}: \delta t=1 \mathrm{~s}(\delta t /\langle\tau\rangle \approx 0.17$ where $\langle\tau\rangle \approx 6.0 \mathrm{~s}), f=10 \mathrm{~Hz}$ and $\delta t=8 \mathrm{~s}(\delta t /\langle\tau\rangle \approx 1.1$ where $<\tau>\approx 7.3 \mathrm{~s}$ ), $f=1.25 \mathrm{~Hz}$. $\tau_{1}$ for the former (red star) and $\tau_{1}$ for the latter (black pentagon) under respective conditions (shown as arrows) are similar, while $\tau_{2}$ for the former (red hexagon) and latter (black diamond) are clearly distinct (shown as arrows), which indicates that $\delta p$ is not a factor to govern the changes in $\tau$ values.

The reason for loss of kinetic heterogeneity is that a t-chip that terminates in a long (short)-dwell-time period of the trace can be incongruously linked to a t-chip that starts in a short (long)-dwell-time period. In the end, the information on kinetic heterogeneity is diluted by this self-averaging process. To avoid such an artifact from the concatenation methods, $\delta t /\langle\tau\rangle$ should be large enough. Our simulations empirically suggest that $\delta t /$ $\langle\tau\rangle$ should be at least 2 to avoid a considerable overestimation of the dwell times (vertical dotted lines in Figure $3 \mathrm{c}-\mathrm{e}$ ). Thus, as a rule of thumb, we suggest that $\delta t /\langle\tau\rangle\rangle 2$ is good for studying kinetic heterogeneity.

The overestimation of $\tau_{1}$ for small $f$ values is due to limited temporal resolution. In this example down to $f=2.5 \mathrm{~Hz}$, the estimates of $\tau_{1}$ are within $30 \%$ of the original $\tau_{1}$. At $f=1.25 \mathrm{~Hz}$, the estimated $\tau_{1}$, however, increases by $\sim 70 \%$ from the original $\tau_{1}$. This augmentation of $\tau_{1}$ is reminiscent of that of $\langle\tau\rangle$ shown in Figure $2 b$, which indicates that the low sampling frequency indeed degrades the accuracy of $\tau_{1}$ as well. Besides this, the overestimation of $\tau_{1}$ should be partly caused by the incongruous linking mentioned before: if a short-lived state is linked to a long-lived state, the overestimation of $\tau_{1}$ would be inevitable. For $\tau_{2}$, its retrieved value can also get longer at low $f$ : if one long-lived high-FRET state is interrupted by a short-lived low-FRET state and the sampling rate is low enough to miss the short state, the long-lived state continues to the next highFRET state beyond the short state without interruption. In that case, a state with the longer dwell time is interpreted to last longer than it does.

Application of the Analysis to Real Problems. In real situations, $\delta t$ is the average time taken from the beginning of measurement until a dye photobleaches and $f$ is determined by the instrumental setting. If the CCD frequency is $10 \mathrm{~Hz}$ and the mean lifetime of fluorescent dye is $300 \mathrm{~s}(=\delta t)$, their product is $3000(=\delta p)$. The concatenation methods including sHaRPer, in general, are useful for a system exhibiting slow kinetics. It is not uncommon to encounter a system with an average dwell time longer than, say, $\langle\tau\rangle_{\text {orig }} \approx 50 \mathrm{~s}$ or more. Thus, $\langle\tau\rangle$ obtained by sHaRPer under that circumstance should be close to $\langle\tau\rangle_{\text {orig }}$ (Figure 2). Sharing benefits and simplicity with other concatenation methods, sHaRPer can be also used to precisely determine $\langle\tau\rangle$ even for small $\delta p$. In the case of extremely small $\delta p$ (for instance, oxygen scavenging methods are not allowed to use), the sHaRPer method would be particularly useful. Thus, regardless of whether $\delta p$ is small or large, we resort to sHaRPer for our discussion.

Next, we ask whether each $\tau$ obtained with sHaRPer is reliable in the presence of kinetic heterogeneity. In the case mentioned previously, $\delta t /\langle\tau\rangle=6(>2)$, and thus $\tau$ values obtained this way should be accurate (Figure $3 c-e$ ). If $\langle\tau\rangle$ becomes larger than $150 \mathrm{~s}, \delta t /<\tau\rangle$ becomes $<2$. In this case, we should be cautious for the possibility that the $\tau$ values obtained as such begin to deviate from the "true" values. Even in such a case, still we can see kinetic heterogeneity: for i-motif, $\langle\tau\rangle \approx$
$300 \mathrm{~s}$, and still we can "resolve" three different rate constants from the survival probabilities of folded and unfolded states of i-motif. ${ }^{10}$ Transitions with a short dwell time are observed multiple times in one measurement duration, permitting a reliable evaluation of the shortest dwell time. Despite the risk of overestimation of $\tau$, we can capture kinetic heterogeneity reliably.

We checked other groups' works from literature to see whether their data can be benefitted by the sHaRPer method (provided that they have a sufficient number of independent traces): (i) Steiner et al. studied slow kinetics of group II intron ribozymes with smFRET. ${ }^{17}$ From data therein, we assume that $<\delta t>\approx 150 \mathrm{~s}$ (from the length of FRET time trajectory) and $f=$ $10 \mathrm{~Hz}$ (not shown; typical value for most FRET measurements). In the work, they estimated $\langle\tau\rangle_{\text {obs }} \approx 42 \mathrm{~s}$, which indicates that the ribozyme indeed exhibits slow kinetics. If we apply the sHaRPer method to get $\langle\tau\rangle$, the estimate should be quite reliable because $f \times\langle\tau\rangle_{\text {obs }} \approx 420(>4)$. If the ribozyme has kinetic heterogeneity, which is assumed to be similar to our simulation example (for instance, $\tau_{2} / \tau_{1}>5$ ), such a kinetic heterogeneity can be also clearly distinguished as $\langle\delta t\rangle \mid\langle\tau\rangle_{\text {obs }}=$ $3.6(>2)$. (ii) Lee et al. studied conformational diversity of human telomeric DNA with smFRET. ${ }^{18}$ We also assume that $<\delta t>\approx 800 \mathrm{~s}$ from the length of FRET time trajectory and $f=$ $10 \mathrm{~Hz}$ (unspecified). In the work, they observed that the DNA molecules exhibited two transition times $\left(\tau_{1}=20, \tau_{2}=188 \mathrm{~s}\right)$ as they managed to measure FRET signals for a long time. sHaRPer can also measure this kinetic information. $\langle\tau\rangle$ can be measured reliably because $f \times \tau_{1}=200(>4)$ and kinetic heterogeneity can be also measured reliably because $\langle\delta t\rangle / \tau_{2} \sim$ $800 / 188 \sim 4(>2)$.

\section{CONCLUSIONS}

In this report, we introduced the sHaRPer method as a means to analyze kinetics of a system whose dwell times of states are long and comparable to the lifetime of fluorescent dye molecules. We showed that the sHaRPer method is a simple and viable means to obtain kinetic information from systems with slow kinetics. The method yields correct average dwell times even in extremely small $\delta p$ or $\delta t$ as long as the data acquisition rate $f$ is sufficiently high. As a rule of thumb, $f$ should be 4 times larger than the average transition rate $\left(f>4\langle\tau\rangle^{-1}\right)$. Although sHaRPer is a universal remedy for average dwell time, it can still degrade the information on, if any, kinetic heterogeneity if $\delta t$ is too short. As a rule of thumb, if $\delta t$ is 2 times greater than the average dwell time $(\delta t>2\langle\tau\rangle)$ so that one can observe on average a few transitions in time traces, $\langle\delta t\rangle /\langle\tau\rangle>\mathrm{O}(1)$, and the data acquisition should at least be made more frequently than the rate of conformational transition of the fastest phase, $f\left\langle\tau_{1}\right\rangle>\mathrm{O}(1)$, sHaRPer can preserve the kinetic heterogeneity of the system, enabling us to obtain the correct time constant of each kinetic component. In practice, data noise even makes an assignment of individual FRET state difficult. In this case, a statistical technique such as hidden Markov modeling, ${ }^{19}$ based on maximum likelihood estimation, can filter the noise, and the resultant time traces can be used as input data for further analysis using sHaRPer. Taken together, as long as one abides by the criteria proposed here in terms of $\langle\delta t\rangle, f$, and $\langle\tau\rangle$, one can use the sHaRPer method and retrieve the kinetic information accurately from a series of short single-molecule time traces. 


\section{ASSOCIATED CONTENT}

\section{S Supporting Information}

Procedures to acquire 2D map of $\langle\tau\rangle \mid\langle\tau\rangle_{\text {orig }}$ from sHaRPer and to obtain the survival probability of a state. Details of how to get Figure $3 \mathrm{~b}-\mathrm{e}$ including simulation conditions and survival probability results from the original traces and traces produced by sHaRPer. The Supporting Information is available free of charge on the ACS Publications website at DOI: 10.1021/ acs.jpcb.5b03991.

\section{AUTHOR INFORMATION}

\section{Corresponding Author}

*E-mail: hongsc@korea.ac.kr.

\section{Notes}

The authors declare no competing financial interest.

\section{ACKNOWLEDGMENTS}

This research was supported by Basic Science Research Program through the National Research Foundation of Korea (NRF) funded by the Ministry of Science, ICT \& Future Planning (2010-00-10594 and 2012R1A1A2021736).

\section{REFERENCES}

(1) Zhuang, X.; Bartley, L. E.; Babcock, H. P.; Russell, R.; Ha, T.; Herschlag, D.; Chu, S. A Single-Molecule Study of RNA Catalysis and Folding. Science 2000, 288, 2048-2051.

(2) Zhuang, X.; Kim, H.; Pereira, M. J.; Babcock, H. P.; Walter, N. G.; Chu, S. Correlating Structural Dynamics and Function in Single Ribozyme Molecules. Science 2002, 296, 1473-1476.

(3) Rhoades, E.; Gussakovsky, E.; Haran, G. Watching Proteins Fold One Molecule at a Time. Proc. Natl. Acad. Sci. U. S. A. 2003, 100, 3197-3202.

(4) Schuler, B.; Eaton, W. A. Protein Folding Studied by SingleMolecule FRET. Curr. Opin. Struct. Biol. 2008, 18, 16-26.

(5) Qu, X.; Smith, G. J.; Lee, K. T.; Sosnick, T. R.; Pan, T.; Scherer, N. F. Single-Molecule Nonequilibrium Periodic $\mathrm{Mg}^{2+}$-Concentration Jump Experiments Reveal Details of the Early Folding Pathways of a Large RNA. Proc. Natl. Acad. Sci. U. S. A. 2008, 105, 6602-6607.

(6) Kalinin, S.; Peulen, T.; Sindbert, S.; Rothwell, P. J.; Berger, S.; Restle, T.; Goody, R. S.; Gohlke, H.; Seidel, C. A. A Toolkit and Benchmark Study for FRET-Restrained High-Precision Structural Modeling. Nat. Methods 2012, 9, 1218-1225.

(7) Selvin, P. R.; Ha, T. Single-Molecule Techniques; Cold Spring Harbor Laboratory Press: New York, 2008.

(8) Swoboda, M.; Henig, J. r.; Cheng, H.-M.; Brugger, D.; Haltrich, D.; Plumeré, N.; Schlierf, M. Enzymatic Oxygen Scavenging for Photostability without pH Drop in Single-Molecule Experiments. ACS Nano 2012, 6, 6364-6369.

(9) Rasnik, I.; McKinney, S. A.; Ha, T. Nonblinking and LongLasting Single-Molecule Fluorescence Imaging. Nat. Methods 2006, 3, 891-893.

(10) Kim, S. E.; Lee, I.-B.; Hyeon, C.; Hong, S.-C. Destabilization of i-Motif by Submolar Concentrations of a Monovalent Cation. J. Phys. Chem. B 2014, 118, 4753-4760.

(11) Lee, I.-B.; Hong, S.-C.; Lee, N.-K.; Johner, A. Kinetics of the Triplex-Duplex Transition in DNA. Biophys. J. 2012, 103, 2492-2501.

(12) Noé, F.; Schütte, C.; Vanden-Eijnden, E.; Reich, L.; Weikl, T. R. Constructing the Equilibrium Ensemble of Folding Pathways From Short Off-Equilibrium Simulations. Proc. Natl. Acad. Sci. U. S. A. 2009, 106, 19011-19016.

(13) Hyeon, C.; Lee, J.; Yoon, J.; Hohng, S.; Thirumalai, D. Hidden Complexity in the Isomerization Dynamics of Holliday Junctions. Nat. Chem. 2012, 4, 907-914.

(14) Thirumalai, D.; Woodson, S. Kinetics of Folding of Proteins and RNA. Acc. Chem. Res. 1996, 29, 433-439.
(15) Pan, J.; Thirumalai, D.; Woodson, S. A. Magnesium-Dependent Folding of Self-Splicing RNA: Exploring the Link between Cooperativity, Thermodynamics, and Kinetics. Proc. Natl. Acad. Sci. U. S. A. 1999, 96, 6149-6154.

(16) Zwanzig, R. Nonequilibrium Statistical Mechanics; Oxford University Press: New York, 2001.

(17) Steiner, M.; Karunatilaka, K. S.; Sigel, R. K.; Rueda, D. SingleMolecule Studies of Group II Intron Ribozymes. Proc. Natl. Acad. Sci. U. S. A. 2008, 105, 13853-13858.

(18) Lee, J.; Okumus, B.; Kim, D.; Ha, T. Extreme Conformational Diversity in Human Telomeric DNA. Proc. Natl. Acad. Sci. U. S. A. 2005, 102, 18938-18943.

(19) McKinney, S. A.; Joo, C.; Ha, T. Analysis of Single-Molecule FRET Trajectories Using Hidden Markov Modeling. Biophys. J. 2006, 91, 1941-1951. 